\title{
GAMMA-RAY BURSTS
}




\title{
Gamma-ray Bursts: Observational Overview, Searches for Counterparts, and BACODINE
}

\author{
G.J. Fishman ${ }^{1}$, S. Barthelmy ${ }^{2}$ \\ 1 Space Sciences Laboratory, NASA/MSFC, Huntsville, AL 35812 USA \\ 2 Laboratory for High Energy Astrophysics, NASA/GSFC, Greenbelt, MD 20771 USA
}

\begin{abstract}
The basic properties of $\gamma$-ray bursts are reviewed. Many of these properties have been known for over two decades, although new and more detailed observations have been made by Compton Observatory in the past three years. The new observation with the greatest impact has been the observed isotropic distribution of bursts along with a deficiency of weak bursts. This is not compatible with any known Galactic population of objects.

The observation of a $\gamma$-ray burst counterpart in another wavelength region has yet to be made, even though there have been substantial efforts in this pursuit from a wide variety of observations in many wavelength regions. Perhaps the greatest chance for a simultaneous or rapid observation of a $\gamma$-ray burst counterpart lies with the newly-developed BAtse COordinates DIstribution NEtwork (BACODINE) system. The objectives and characteristics of this system are described.
\end{abstract}

\section{Introduction}

It is over 25 years since the discovery of $\gamma$-ray bursts, and their origin appears as elusive as ever. The observed isotropy and inhomogeneity of these objects represent a distribution unlike any other known galactic objects. Over a hundred theories on their origin have now been cataloged (Nemiroff 1994). These models cover distance scales from the Oort cloud to cosmological distances. Never before in modern astronomy has there been so great an uncertainty in the distance to a class of objects. There is a similar level of uncertainty about the basic nature of the underlying source and the emission mechanism. Whatever the distance scale and the source of the bursts, it will most likely represent a new class of objects, processes and/or emission mechanism.

In the first section, this paper summarizes the basic observed properties of $\gamma$-ray bursts, derived from lectures presented at the Les Houches School for Theoretical Physics in January 1994. Later sections describe past and present attempts to observe counterparts of $\gamma$-ray bursts in another wavelength region and the newly-developed BACODINE system. The objectives and characteristics of this system offers the unprecedented opportunity to search for $\gamma$-ray burst counterparts in near-realtime. 


\section{Observed properties of $\gamma$-ray bursts}

Considerable observational progress has been made in the past few years as more sensitive space-borne detectors have become available. Many of the observations have been made by the Burst and Transient Source Experiment (BATSE) on the Compton Gamma-Ray Observatory ( $C G R O)$. While most of these observational results are relatively straight-forward, some of the properties and interpretations of ensembles of bursts are the subject of analyses that are often debatable. Comprehensive sets of papers of the more recent observational results can be found in conference proceedings that have been published in the past three years (Paciesas \& Fishman 1992, Friedlander et al. 1993, Fishman et al. 1994). Hartmann (1995) also provides a detailed list of $\gamma$-ray burst (GRB) reviews and Hurley (1994) has made available a comprehensive bibliography of the GRB literature.

Perhaps the most striking features of the time profiles of GRBs are their morphological diversity and the large range of burst durations. A cursory examination of burst profiles indicates that some are chaotic and spiky with large fluctuations on all timescales, while others show rather simple structures with few peaks. However, some bursts are seen with both characteristics present within the same burst. No periodic structures have been seen from GRBs. Coupled with this diversity is the general inability to place many GRBs into well-defined classifications. Examples of extreme differences in burst morphologies and durations are shown in a sample page from the First BATSE Burst Catalog (Fishman et al. 1994; Fig. 1).

The durations of $\gamma$-ray bursts range from about $10 \mathrm{~ms}$ to over $1000 \mathrm{~s}$ in the energy range in which most bursts are observed. A bi-modality is seen in the logarithmic distribution with broad, un-resolved peaks at about $0.3 \mathrm{~s}$ and 20 $s$ and a minimum at around two seconds (Fig. 2). The shorter bursts are also seen to have harder spectra, as measured by a hardness ratio (Kouveliotou et al. 1994). Sub-millisecond structure has been detected in at least one burst (Bhat et al. 1992). Recent EGRET-CGRO observations show high energy (>100 MeV) emission over 90 minutes after the burst trigger (Hurley et al. 1994). Another general property of the $\gamma$-ray bursts time profiles is that they tend to have shorter rise-times and fall-times (sharper spikes) at higher energies. Most bursts also show an asymmetry, with shorter leading edges than trailing edges. This has been quantified (Link et al. 1993, Nemiroff et al. 1994).

An analysis of time profiles that could have extraordinary consequences is the observation by Norris et al. (1994) of a systematic widening or stretching of $\gamma$ ray burst time profiles as bursts become weaker. This analysis was performed by artificially weakening the stronger $\gamma$-ray bursts and introducing the appropriate background so that all bursts could be analyzed in a consistent manner. The observed stretching of the profiles of bursts is consistent with that expected from the effects of time-dilation from bursts at cosmological distances. However, this observation and its interpretation are not universally accepted.

Another distinguishing feature of $\gamma$-ray bursts is their high-energy emission: almost all of the power is emitted above $50 \mathrm{keV}$. Some bursts show emission as 

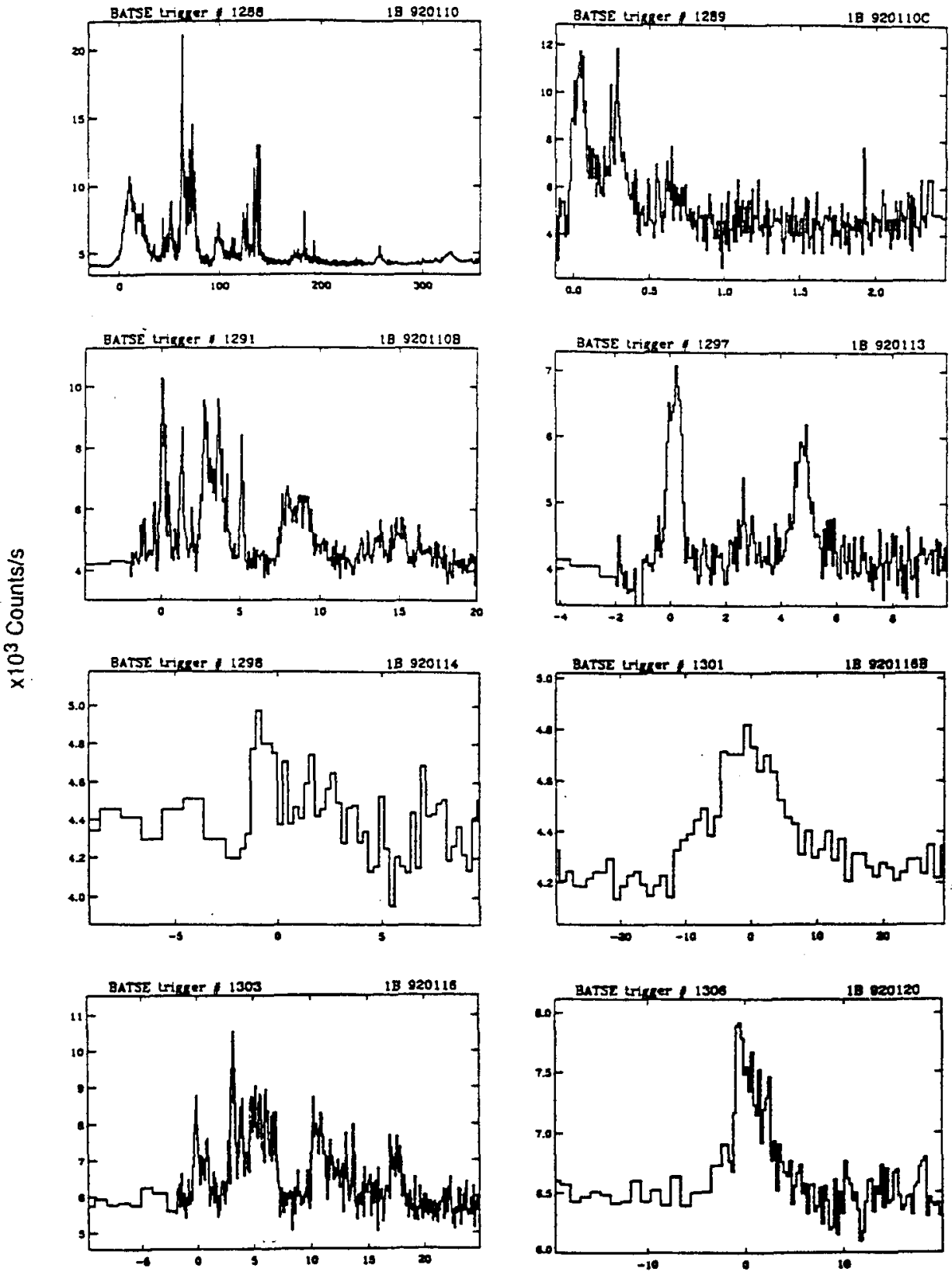

Seconds (Rel. to Trigger)

Fig. 1. A sample of eight $\gamma$-ray bursts for the First BATSE Catalog (Fishman et al. 1994), showing the extreme range of burst time profiles and durations. 


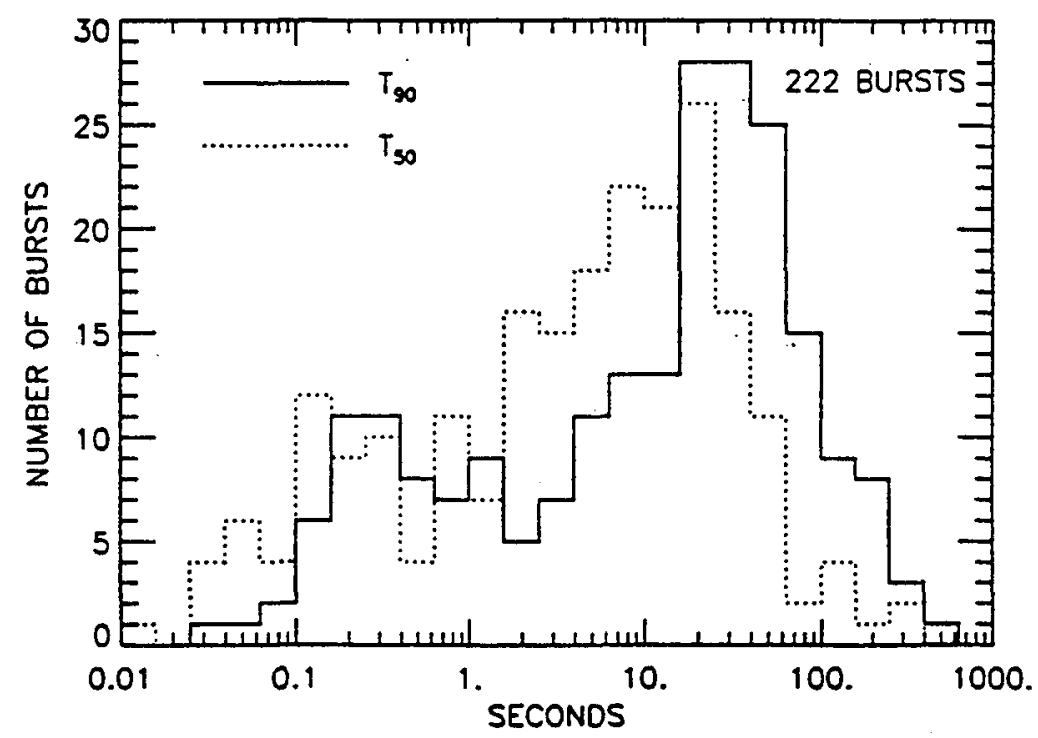

Fig. 2. The duration distribution of $\gamma$-ray bursts from the First BATSE Burst Catalog (Fishman et al. 1994).

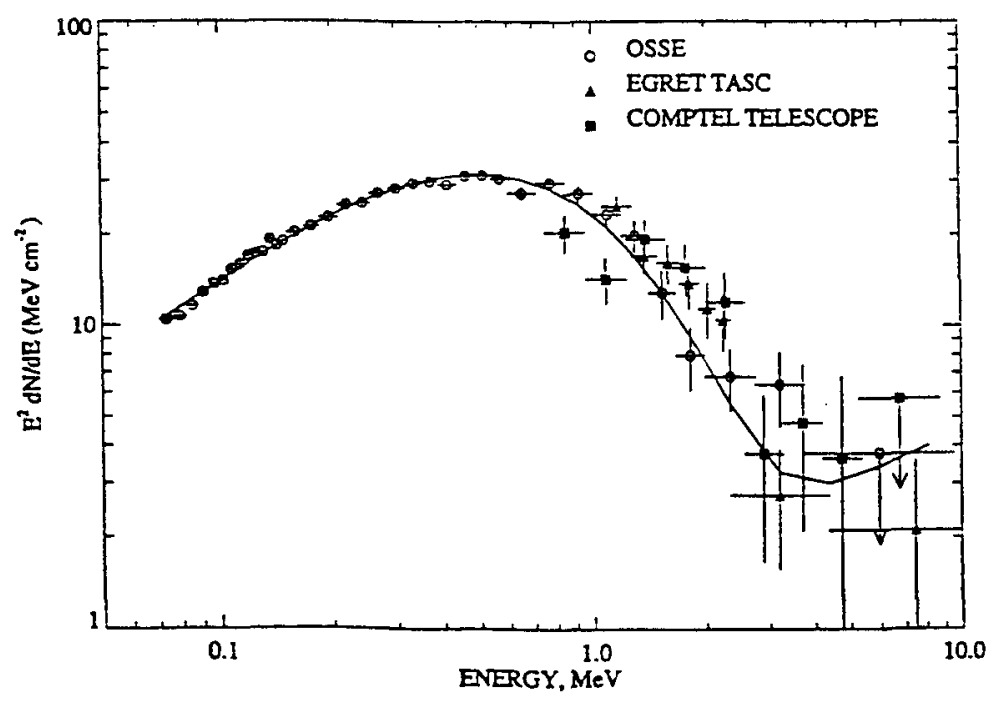

Fig. 3. The high energy spectrum from GB 910601, as measured by three of the experiments on the Compton Observatory (Share et al. 1994), integrated over a large portion of the burst. A characteristic broad spectral shape, with peak power of about $0.6 \mathrm{MeV}$ is seen. (The spectral up-turn at high energies is not real.) 
low as $1 \mathrm{keV}$, but the power is less than 1 or $2 \%$ of the total power (Yoshida et al. 1989). Most bursts show rather simple continua spectra which appear similar in shape when integrated over the entire burst and when sampled on various timescales within a burst. Fig. 3 shows a typical burst spectrum from $0.1-10$ $\mathrm{MeV}$, with the peak power at about $600 \mathrm{keV}$ (Share et al. 1994). Spectral shapes which have been fit to burst spectra include broken power laws (Schaefer et al. 1992), log-normal distributions (Pendleton et al. 1994), and exponential spectra with power-law high energy tails (Band et al. 1993). Although the spectral shapes of many bursts are similar, the energy at which peak power is emitted changes greatly from burst-to-burst and rapidly within a burst. Some significant changes on time scales as short as tens of milliseconds have been observed (cf. Ford et al. 1994). In the past few years, EGRET-GRO has seen significant flux and power into the $\mathrm{GeV}$ energy range from several bursts. A very strong burst with high energy emission was detected on 17 February 1994 Hurley et al. (1994). Many of the high energy photons are delayed with respect to the bulk of the lower energy emission. There was no observed emission at lower energies from the burst region at that time, as observed by the BATSE experiment. A single $20 \mathrm{GeV}$ photon was recorded from a burst direction which is the highest energy ever recorded from a GRB.

A search for unambiguous $\gamma$-ray line features with $B A T S E / G R O$ has thus far been unable to confirm the earlier reports of spectral line features from $\gamma$-ray bursts. Several papers from the 1993 Huntsville gamma-ray burst workshop proceedings (Fishman et al. 1994) and Palmer et al. (1994) discuss the preliminary $B A T S E$ line search analyses and their results. This work is still in progress.

The isotropy of the BATSE $\gamma$-ray burst distribution (Fig. 4), coupled with its inhomogeneity (as measured by the deficiency of weak $\gamma$-ray bursts) continues to be the most surprising recent observation of GRBs, and the one that has eliminated most of the expected and reasonable Galactic distribution models. The BATSE intensity distribution has been combined with the PVO intensity distribution to yield a combined data set of almost four decades in intensity (Fenimore et al. 1993). This composite intensity distribution matches well in the overlap region, showing a smooth transition to the $-3 / 2$ power law expected from a homogeneous distribution at the higher intensities and a clear deviation from this homogeneous distribution at low intensities.

The three distribution models most often discussed as being compatible with the observation of isotropy and inhomogeneity are: (1) An extended solar system model such as an Oort cloud or other sun-centered large distribution (cf. White 1993, 1994); (2) An extended Galactic halo or Galactic corona model, and (3) A cosmological model. A recent paper by Hakkila et al. (1994) shows that the parameter space available to the extended Galactic models is shrinking to unrealistic values, e.g. typical source distances of $70 \mathrm{kpc}$. Many believe that solar-system based models would likewise show an observable anisotropy. Thus, from the isotropy observations alone, one is increasingly forced to think in terms of cosmological models. 


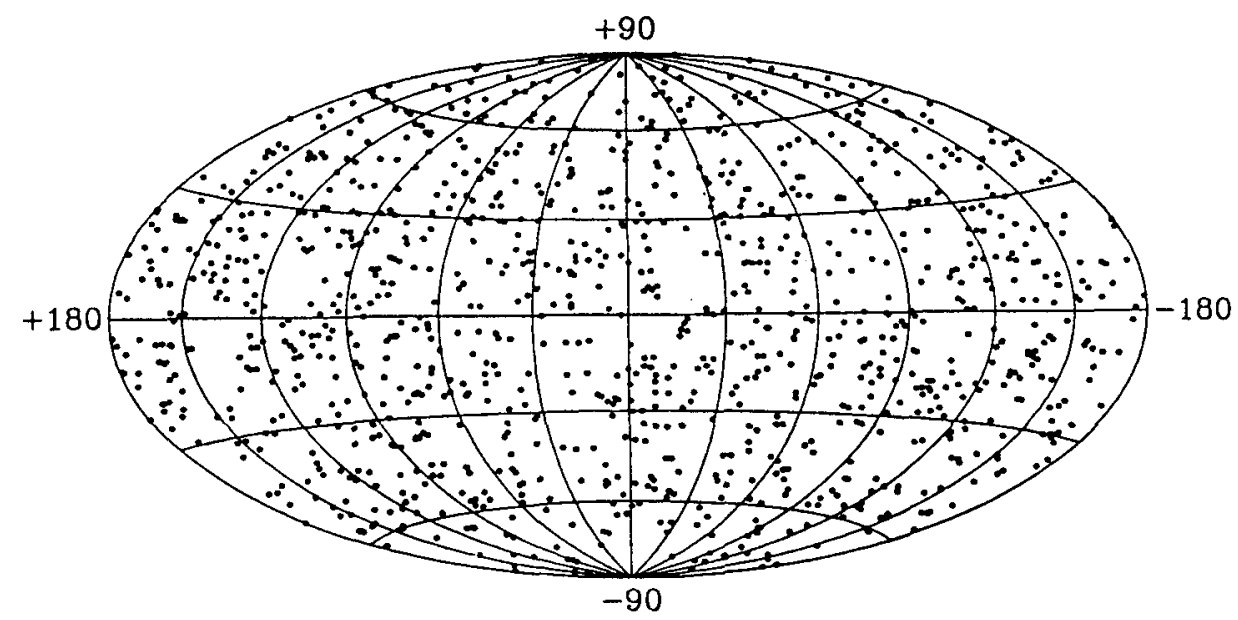

Galactic Coordinates

Fig. 4. The distribution of 921 GRBs observed by BATSE on CGRO in Galactic coordinates. More accurate burst locations will become available as the data are further processed for subsequent catalogs. The isotropy of the bursts is apparent.

\section{Burst Counterparts}

There is no doubt that a great advance in our understanding of GRBs can be attained through successful correlated observations of GRBs at other wavelengths. This fact was demonstrated recently by the combined $\gamma$-ray, X-ray, optical and radio observations of Soft $\gamma$-ray Repeaters (SGR's) (Kouveliotou et al. 1994, Murakami et al. 1994, Kulkarni et al. 1994). Within the past three years, there have been major, renewed efforts to find a counterpart to a GRB in other wavelength regions as evidenced by either simultaneous emission or afterglow emission. Comprehensive studies of archival plates also have been made. There have been several suggestions for counterparts although the results are problematic and are often debated. In view of the importance of the implied results, further observational evidence is needed before these results are accepted. Some of the world's most powerful ground-based facilities for radio and optical astronomy, high-energy air showers, atmospheric C̈erenkov, and neutrino and gravitational wave astronomy are participating in these attempts for correlated burst observations. Space-borne correlated observations of well-located GRBs have also been attempted in the UV, EUV, and X-ray regions. A recent review of the present status of correlated GRB observations is given by Schaefer (1994).

Two different strategies have been employed to make follow-up observations to identify the GRB source object in the optical and other band passes. The first method is to view a fixed point in the sky in the hopes that a GRB will occur in the FoV. Because it is impossible to predict when and where a GRB will occur, very large fields-of-view (FoV) are required for the chance "observing" of 
a GRB. Because, in general, the large FoVs result in poor spatial resolution and crowded fields at the detector, this method has poor sensitivity.

The second method improves the sensitivity by limiting the FoV at the expense of making the observation with some time delay after the GRB. By waiting until after the GRB, rough positional information from instruments in orbit (e.g. GRO-BATSE/COMPTEL, WATCH-GRANAT/EURECA, IPN) can be obtained to direct narrower FoV instruments to the GRB error box. While the time delays between the GRBs and the observations have been steadily improving over the years - currently they are down to 5-to-36 hours range, still no positive identification of a GRB source object has been made. The general conclusion of these negative observations is that the optical emission from the source objects must have faded below $\approx 20$ th magnitude within several hours.

Since detector technology is unlikely to improve in the next few years to the point where the first method (stare mode) is likely to yield any new or different results, attempts are being made to improve the second method (follow-up mode). Clearly, the desired goal is to obtain accurate positional information on the GRB and to quickly get that information to instruments capable of making rapid follow-up observations.

The long-baseline interplanetary network (IPN) of burst detectors have provided the most accurate locations for these correlated observations. With the loss of both the PVO and Mars Observer spacecraft in recent years, the detectors aboard the Ulysses spacecraft remain the only remote, long baseline component that can be used with earth-orbiting detectors. The narrow (usually a few arcminutes) annuli derived from the Ulysses-Earth network observations can also be used in conjunction with coarser, single-spacecraft locations to greatly restrict the error box of some bursts. This situation will persist until the Russian Mars 96 mission is launched. For non-IPN localizations, the COMPTEL and $E G R E T$ on the Compton Observatory can provide burst location accuracies of the order of one degree, for strong bursts with high-energy emission which happen to be in their field-of-view. SIGMA-GRANAT and WATCH-GRANAT also continue to provide accurate locations for accessible bursts. Those ground-based searches that can utilize the rather coarse $B A T S E$-derived burst locations $\left(\approx 4^{\circ}\right.$ for intense bursts) such as wide field Schmidt cameras, can also respond to these bursts quicker than ever before possible.

Since 1991, BATSE has had a quick alert capability that was developed to provide burst locations within several hours, under favorable conditions. A joint BATSE-COMPTEL capability also exists that is able to provide even more accurate $\left(\approx 1^{\circ}\right)$ locations within several hours for those GRBs which also happen to be within the COMPTEL FoV. This capability was demonstrated for the intense GRB of 31 January 1993 (the Superbowl Burst), when an extraordinary effort involving over 30 instruments observed the burst region within hours and days of its occurrence (Schaefer et al. 1994). A sensitive, wide-field transient optical camera has also been operating for over three years at Kitt Peak (Vanderspek et al. 1994), responding to BATSE-triggered bursts. 


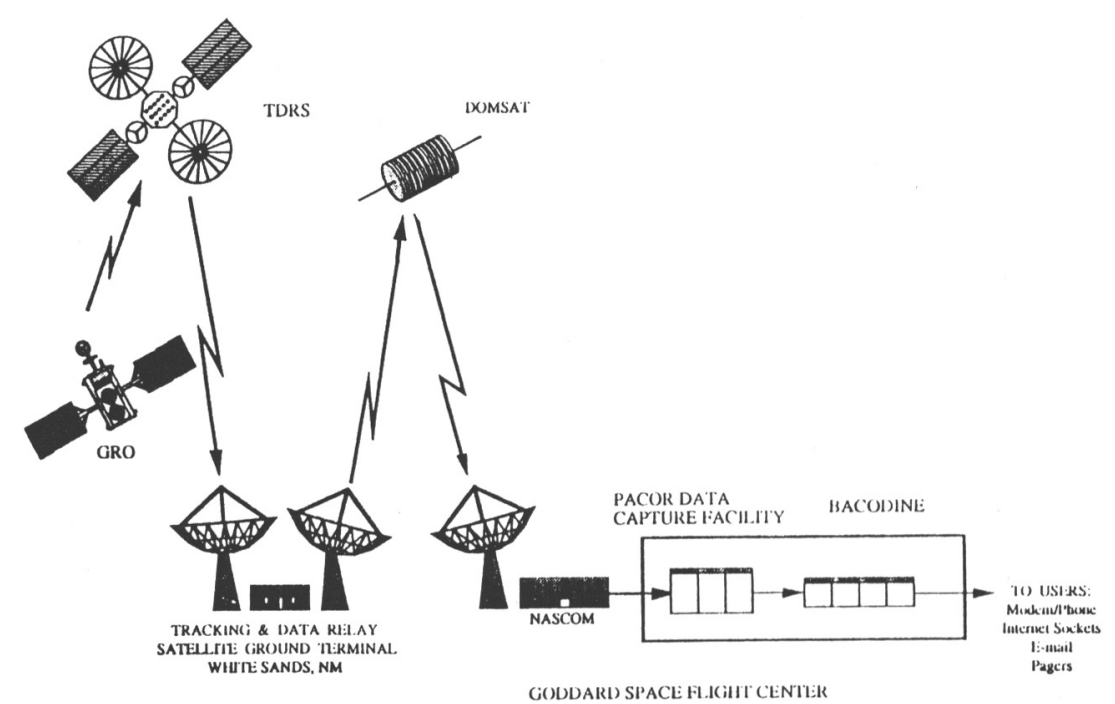

Fig. 5. Schematic diagram showing the various facilities and transmission paths and time delays in the $B A C O D I N E$ system.

\section{The BACODINE System}

A new, near-realtime $B A T S E$ burst location system called BACODINE (Barthelmy et al. 1994) became operational in 1993. This system, when linked to a rapid-slewing optical telescope, opens the exciting possibility of obtaining optical images of burst regions while the burst is in progress. Since the failure of the on-board tape recorders on $C G R O$, real-time transmission of the data from the spacecraft now allows for nearly continuous, real-time access to the BATSE data. These data can be used to make simultaneous or near-simultaneous multiband observations of GRBs. The BACODINE system: (1) monitors the CGRO telemetry stream, (2) extracts the appropriate information from the BATSE portion of the data, (3) detects the occurrence of a GRB, (4) calculates the approximate coordinates for the burst, and (5) distributes those coordinates to observatories, other spacecraft and other interested parties around the world. This is done with custom hardware and software located at the Goddard Space Flight Center (GSFC) mission operations center for CGRO.

For those portions of the orbit where $C G R O$ can get direct line-of-sight transmission of its high-gain antenna to any of the three TDRS satellites, the data are relayed to the NASA White Sands Ground Station in New Mexico where they are retransmitted to a domestic communications satellite (DOMSAT) and then transmitted back down to the GSFC Data Capture Facility (Fig. 5). There is an additional 1.0 seconds of time delay due to four hops of ground-to-geosync-orbit light-travel time and buffering within the White Sands facility. Once received at GSFC, the entire $2.048 \mathrm{sec}$ of data is processed to yield GRB coordinates within $0.1 \mathrm{sec}$. The fastest method of coordinate distribution (see below) takes an ad- 
ditional $0.3 \mathrm{sec}$. If the GRB started during the beginning of the first 1.024-sec count-rate sample, then the total time delay between when the $\gamma$ rays interacted with the BATSE LADs and when the coordinates are available at an instrument to make follow-up observations is $5.50 \mathrm{sec}$. If the GRB started at the end of the second 1.024-sec sample, then the time delay is $3.45 \mathrm{sec}$. More than half of the GRBs are longer than $5.5 \mathrm{sec}$, thus allowing follow-up observations to be made while the burst is still occurring.

Currently, all data from $G R O$ is transmitted to ground in real-time (with essentially no time delay) with about $85 \%$ live-time. BATSE detects about 0.8 GRBs per day. Using the typical ground-based observational efficiencies encountered, the rate of making follow-up observations is $0.063 \mathrm{GRBs}$ per day or once every 16 nights. If factors for new moon, for the brightest and for the longest GRBs are included; then the rate is 0.008 per day or once every 4.2 months.

The sequence of the processing of data in $B A C O D I N E$ can be broken down into five general steps: (1) The program monitors the telemetry stream continuously extracting the count rates for the eight $B A T S E$ detectors, comparing these rates to the calculated average current background rates. (It also extracts some general purpose housekeeping information in the process). (2) It monitors the "burst-in-progress" flag generated by the BATSE flight data processor and, when set true, it (3) takes the current count rates, subtracts the previously accumulated background rates to get the source-only rates, finds the three brightest detectors, and (4) solves the set of 3 simultaneous equations of the dot-product of the unknown burst direction and the detector normals of the three brightest detectors. (5) The burst direction is then sent to a list of instruments at various sites world-wide that are capable of making follow-up observations. Currently, the algorithm used to calculate the GRB direction assumes "ideal response" for the BATSE detectors. This approximation yields an uncertainty for the $B A C O$ $D I N E$ burst position of about a $20^{\circ}$ diameter error circle.

There are six possible methods for distributing the BACODINE GRB coordinates. The fastest method is the dedicated phone line. Around sunset at the instrument site (assuming it is an optical instrument), a phone/modem connection is made between the BACODINE computer and the computer at the instrument site. This connection is maintained throughout the night and should a burst occur during this time the coordinates (RA, Dec) are sent over the connection. At 9600 baud it takes 0.3 seconds. The second fastest method, and much less costly, is the Internet socket connection. Sockets is a technique to connect two programs running on two computers over a network. Like the dedicated phone method, the socket connection is made at some initial time and then maintained indefinitely. The time delay for the propagation of the coordinates packet varies due to the distance between the two computers, the number of routers and gateways in use, and the amount of other network traffic. However, it has been routinely shown that for a connection between Maryland and California (coast to coast US) the round-trip propagation time is 0.8 seconds average and 2.0 seconds maximum (1.2 and $5 \mathrm{sec}$, respectively, for Cambridge, England). It is difficult to make one-way travel time measurements on the Inter- 


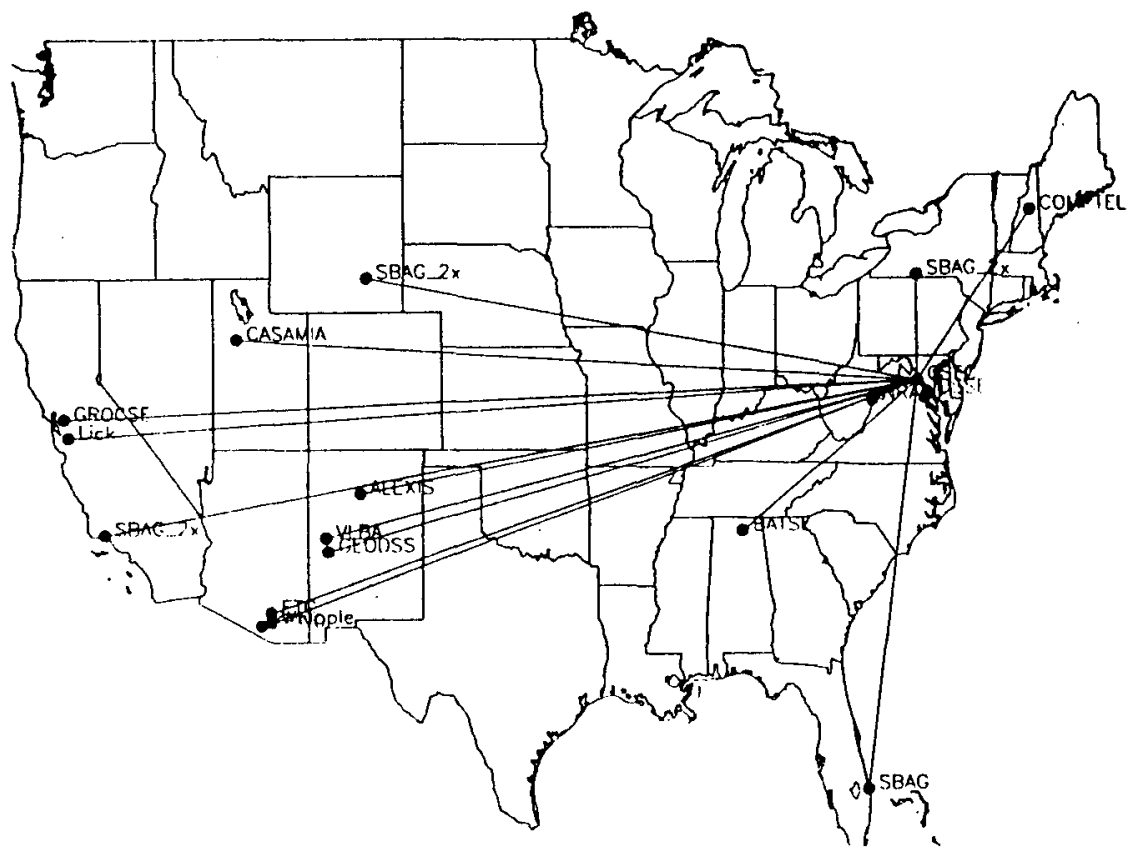

Fig. 6. A map of the US, showing the facilities that are presently receiving and responding to the $B A C O D I N E$-distributed signals.

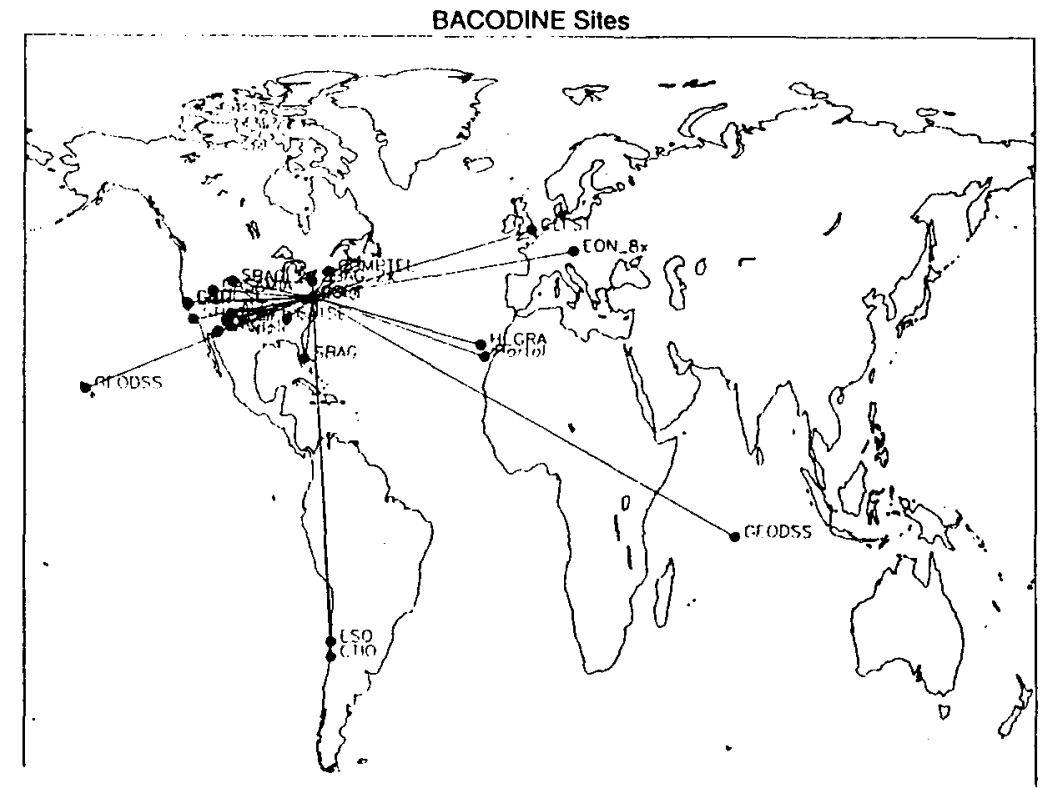

Fig. 7. World map showing the locations of BACODINE collaborating sites. 
net, so we use round-trip travel times and assume that the one-way time is half the round-trip time. The e-mail method is relatively fast and suitable for sites with non-automated instruments (i.e. humans in the loop). The alpha-numeric pagers work well for sites without phone or Internet connections. The pager displays the RA, Dec location, the time and the initial intensity of the GRB. The pager companies accept the $B A C O D I N E$ notices via an Internet e-mail address and transmit the message to the designated pager unit. The entire process takes 1 to 2 minutes.

Currently, there are 21 groups collaborating with $B A C O D I N E$ at 34 sites around the world. Figs. 6 and 7 show US and world maps designating the locations of these ground-based instruments. Of the 21 groups, eight have optical instruments, four are radio, and three are in the very high-energy ( $\mathrm{TeV} / \mathrm{PeV})$ $\gamma$-ray region. Of particular interest are several automated instruments which are fully computer-controlled and have rapid response and fast slewing times. Thus, they have the shortest delay time ( 5 to $30 \mathrm{sec}$ range) to make an observation of a GRB error box. They are the LLNL GROCSE optical instrument in Livermore, CA; the MIT ETC optical instrument at Kitt Peak, AZ; and the CLFST radio instrument at Cambridge, England.

In addition to the normal operations with instruments that can provide rapid follow-up observations, $B A C O D I N E$ also provides rapid notification to other groups that are active in the GRB field (e.g. K. Hurley et al. for the Interplanetary Network (IPN); J. Block with the $A L E X I S$ spacecraft, and the GRO-OSSE \& GRO-EGRET teams). BACODINE also captures the GROCOMPTEL telemetry data for those GRBs which are in the COMPTEL FoV. The program automatically ftp's the data to a computer at UNH where it is automatically analyzed. If the GRB is bright enough, a $1-2^{\circ}$ COMPTEL location can be obtained in 15-30 minutes. The BACODINE system also has the ability to "filter" or custom the notifications to each site according to several criteria and needs. The basic filtering is a visibility and or night-time requirement. If the site operates in the radio band-pass, then the BACODINE program will send that site a notice only if the calculated GRB location is visible $\left(>10^{\circ}\right.$ above its local horizon). If the site is optical then it also requires that it be night at the site (the sun is more than $6^{\circ}$ below the local horizon). An intensity threshold can also be applied. This threshold is used for instruments which have a small FoV and thus require more accurate locations to be effective.

We would like to encourage the participation of sites at many different longitudes and latitudes (in particular the Southern Hemisphere). We are currently working on improving the location accuracy by several methods. It is believed that the GRB position uncertainty will decrease to perhaps a few degrees radius for a strong burst. In the future, the BACODINE computers and networks will also capture and redistribute transient locations detected by the HETE spacecraft (to be launched in 1995) and by the IPN. Because these two GRB localization sources produce smaller error boxes, more traditional, narrow FoV telescopes with much fainter sensitivity can be used to make follow-up observations. 


\section{Summary}

The GRB enigma appears to be as great now as it was twenty years ago (Ruderman 1975). A wealth of new data on time profiles, spectral characteristics and burst distributions has thus far failed to provided conclusive evidence on the distance scale, central object or emission mechanism for the classical GRBs. The isotropy and inhomogeneity of the bursts only shows that we are at the center of the apparent burst distribution. The recent EGRET-CGRO discovery of delayed $\mathrm{GeV}$ emission from a burst is yet another severe constraint for many of the burst models. Many feel that the identification of a burst with an object in another wavelength region may be the key to understanding these objects. The $B A C O D I N E$ system is now operational and may ultimately yield the long-awaited, unambiguous counterpart to a GRB.

\section{References}

Band D. et al., 1993, ApJ 413, 281

Barthelmy S. et al., 1994, Huntsville GRB Workshop, AIP 307, AIP, New York, p. 643

Bhat P.N., et al., 1992, Nature 359, 219

Fenimore E.E., et al., 1993, Nature 366, 40

Fishman G.J. et al., 1994, ApJS 92, 229

Fishman G.J., Brainerd J.J., Hurley K., 1994, Huntsville GRB Workshop, AIP 307, AIP, New York

Ford L., et al., 1994, ApJ 439, 305

Friedlander M. et al., 1993, Compton Gamma-Ray Observatory, AIP 280, New York

Hakkila J. et al., 1994, ApJ 422, 659

Hartmann D., 1995, The Gamma-ray Sky by SIGMA and CGRO, NATO ASI Proc.

Hurley K., 1994, Huntsville GRB Workshop, AIP 307, AIP, New York, p. 726

Hurley K., et al., 1994, Nature 372, 652

Kouveliotou C. et al., 1993, ApJ 413, L101

Kouveliotou C. et al., 1994, Nature 368, 125

Kulkarni S.R. et al., 1994, Nature 368, 129

Link B., Epstein R.I., Priedhorsky W.C., 1993, ApJ 408, L81

Murakami T. et al., 1994, Nature 368, 127

Nemiroff R.J., 1994, Huntsville GRB Workshop, AIP 307, AIP, New York, p. 730

Nemiroff R.J. et al., 1994, ApJ 423, 432

Norris J. et al., 1994, ApJ 424, 540

Paciesas W.S., Fishman G.J., 1992, Huntsville GRB Workshop, AIP 265, New York

Palmer D., et al., 1994, ApJ 433, L77

Pendleton G.N. et al., 1994, ApJ 431, 416

Ruderman M., 1975, Ann. N.Y. Acad. Sci. 262, 164

Schaefer B.E. et al., 1992, ApJ 393, L51

Schaefer B.E., 1994, Huntsville GRB Workshop, AIP 307, AIP, New York, p. 382

Schaefer B.E. et al., 1994, ApJ 422, L71

Share G. et al., 1994, Huntsville GRB Workshop, AIP 307, AIP, New York, p. 283

Vanderspek R., Krimm H., Ricker G., 1994, Huntsville GRB Workshop, AIP 307, AIP, New York, p. 438

Yoshida A. et al., 1989, PASJ 41, 509

White R.S., 1993, ApSS 208, 301

White R.S., 1994, Huntsville GRB Workshop, AIP 307 AIP, New York, p. 620 